\title{
Review of: "Deactivation blocks proton pathways in the mitochondrial complex I"
}

\author{
Thorsten Friedrich ${ }^{1}$ \\ 1 Albert-Ludwigs-Universität Freiburg
}

Potential competing interests: The author(s) declared that no potential competing interests exist.

Mitochondria are known as power houses of cells because they provide more than $90 \%$ of the energy currency adenosine triphosphate (ATP). The making of ATP takes place in the inner mitochondrial membrane by the action of the enzyme complexes of the respiratory chain that build up a proton gradient. This gradient drives ATP-synthase from ADP plus phosphate. The enzyme complexes of the respiratory chain use energy provided by metabolic substrates such as $\mathrm{NADH}$, succinate, and so on. The substrates are oxidized and the electrons are passed through a series of enzyme complexes finally to oxygen that is reduced to water. The first enzyme complex of the mitochondrial respiratory chain is the energy-converting NADH:ubiquinone oxidoreductase, also called respiratory complex I.

Complex I oxidizes NADH and reduces the mobile electron carrier ubiquinone (Q) coupled with the translocation of 4 protons across the inner mitochondrial membrane. The complex roughly consists of two parts: A so-called peripheral arm catalyzing the electron transfer reaction and a membrane arm involved in proton translocation. Complex I is characterized by an unusual Q binding cavity that is buried deep in the enzyme at the connection between the membrane and the peripheral arm and further by a 'vein' of charged amino acids residues and water molecules lining up in the middle of the membrane arm. It is thought that the chain of titratable residues connects the individual proton channels in the membrane arm of the complex. Remarkably, the reaction of complex I is fully reversible, which means that ubiquinol $\left(\mathrm{QH}_{2}\right)$ is oxidized and $\mathrm{NAD}^{+}$is reduced, a reaction driven by the reverse proton flow across through the proton translocation pathways. This process is called reverse electron transfer (RET). Our understanding of the reaction of the complex and its regulation was hampered by the lack of structural data. However, during the last years structures of the complex from several species became available due to the progress in electron cryo-microscopy (cryo-EM) which greatly accelerated the development in the field.

The reversibility of the enzyme's reaction may become problematic under certain conditions. Under hypoxic or anoxic conditions that might be an outcome from a stroke or tissue damage (such as reperfusion injury), RET in complex I may lead to the generation of reactive oxygen species that are physiologically harmful. It is thought that under low turnover conditions, mammalian complex I can convert to a 'deactive' state (D) that is catalytically inactive. Indeed, structures of the complex in the 'active' (A) 
and the D state were recently presented showing small but distinct differences. It is still under debate whether the $\mathrm{D}$ form of the complex represent an intermediate of the catalytic cycle or whether it represents an 'off-cycle' state.

In their recent PNAS paper, Röpke et al. tackled this question by using a combination of large-scale classical and quantum mechanical simulations based on the recent cryo-EM structural data. They used a model of the mouse mitochondrial complex I in the A state and targeted the structure with data obtained from the $D$ state to a full atomic model of the $D$ state. Due to the resolution limitations in structure determination the model resulted in a 'dry' state that was filled with water molecules during simulations. The two structures differed by a relative twist between the membrane and the peripheral arm as described for the $A$ and the $D$ state by cryo-EM. The structures of both states were stable over the entire simulation time. The most striking changes were seen in the region of the membrane arm that is connected to the peripheral arm. Three subunits, called ND3, ND4L and ND6, showed conformational changes in the arrangement of loops connecting individual transmembranous (TM) helices and especially in TM helix 3 of ND6 that partly turned into a $\pi$-bulge. The conformational changes in that region were also described in cryo-EM structures of a yeast and of mammalian complex I. The structural rearrangement of ND3, ND4L and ND6 had dramatic consequences for the water-mediated proton pathway connecting the Q binding site to the proposed proton channels. A methionine residue that is part of the $\pi$-bulge flips into the watermediated proton pathway disconnecting both sites. Thus, the $\mathrm{Q}$ binding and reduction site is disconnected from the proton translocation pathways in the D state, energy transduction is interrupted.

Placing a Q molecule in the structure, Röpke et al. demonstrated that the transition from the A to the D state also interferes with Q chemistry. The Q molecule has two preferred positions in the binding pocket that were also found in other MD simulations and that agree with positions described by cryo-EM structures. When $\mathrm{Q}$ is bound in the 'lower' position that is closer the P-side of the membrane it may be converted to a quinol anion $\left(\mathrm{QH}^{-}\right)$species as proposed from spectroscopic data. The de-protonation of $\mathrm{QH}_{2}$ leading to the generation of $\mathrm{QH}^{-}$might initiate the protonation cascade along the chain of titratable amino acid residues and water molecules in the membrane arm that would cause loading of the proton channels, while the backward cascade would lead to the release of protons from the channels to the P-side. The connection between the proton transfer cascade and bound $\mathrm{QH}_{2}$ is broken in the $\mathrm{D}$ state so that RET cannot take place because it lacks the energy driving this process provided by the proton translocation from the $\mathrm{P}$ - to the $\mathrm{N}$-side.

The elegant paper by Röpke et al. provides a feasible explanation how the transition from the A- to the Dstate prevents the formation of deleterious reactive oxygen species by respiratory complex I. They propose that the interface of subunits ND3, ND4L and ND6 provide a 'deactivation gate' represents a a fine-tuning device to modulate proton translocation in mammalian complex I. However, further 
experiments and theoretical considerations are needed to clarify whether the $D$ state is part of the reaction cycle of the complex or whether it is an 'off-pathway' state. 